Supporting Information

\title{
A Robust Nitric Oxide Microsensor for High Spatial Resolution Measurements in Biofilms and Sediments
}

Frank Schreiber*, Lubos Polerecky and Dirk de Beer

Microsensor Research Group, Max-Planck-Institute for Marine Microbiology, Celsiusstrasse 1, D28359 Bremen, Germany

\section{Contents}

1) Theoretical maximum of sensitivity for a combined NO-microsensor operating at a constant potential

2) Theoretical maximum of sensitivity for a bare single-anode NO-microsensor operating at constant potential

3) Influence of the guard anode on fluctuations of background currents 


\section{1) Theoretical maximum of sensitivity for a combined NO-microsensor operating at a constant potential}

The theoretically maximum sensitivity for the combined NO microsensor (Fig. 1) is estimated by calculating the maximum flux of NO molecules towards the sensing electrode at a given NO bulk concentration $c_{b u l k}$, assuming that the concentration of the reduced NO molecules at the sensing surface is zero (Fig. S1).

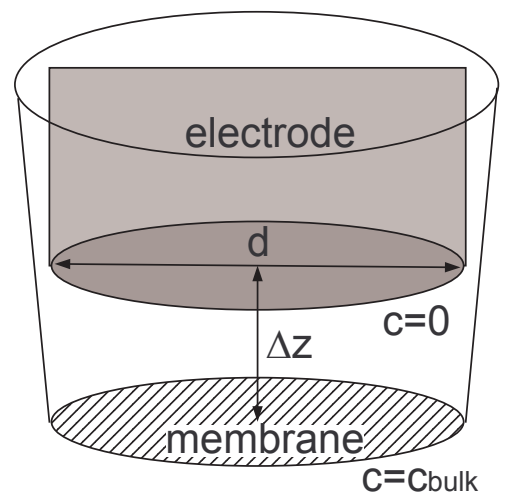

Figure S1:

Molecules that enter the sensor through the membrane are transported by diffusion over a distance $\Delta \mathrm{z}$ to the planar sensing surface (diameter $d$ ). Using Fick's $1^{\text {st }}$ law of diffusion, the flux of the NO molecules is

$$
\mathrm{J}=-\mathrm{D} \Delta \mathrm{c} / \Delta \mathrm{z}
$$

where $\mathrm{D}=2.21 \times 10^{-9} \mathrm{~m}^{2} \mathrm{~s}^{-1}$ is the molecular diffusion coefficient of $\mathrm{NO}$ in the aqueous electrolyte and $\Delta \mathrm{c}$ is the concentration difference between the bulk medium and the sensing surface, i.e., $\Delta \mathrm{c}=\mathrm{c}_{\text {bulk }}$. The 
diffusion of NO through the silicone membrane can be assumed to be equal to that in water because similar diffusion coefficients in both were reported earlier ${ }^{1,2}$. Considering that the reaction of one NO molecule at the sensing surface donates 3 electrons to the measuring circuit $\left(\mathrm{NO}+2 \mathrm{H}_{2} \mathrm{O}-3 \mathrm{e}-\mathrm{NO}_{3}{ }^{-}\right.$ $+4 \mathrm{H}^{+}$), the current through the electrode can be written as

$$
I=3 A J \mathrm{~N}_{\mathrm{A}} \mathrm{C}_{\mathrm{e}-}
$$

where $\mathrm{N}_{\mathrm{A}}=6.022 \times 10^{23} \mathrm{~mol}^{-1}$ is Avogadro's constant, $\mathrm{C}_{\mathrm{e}}=-1.6022 \times 10^{-19} \mathrm{C}$ is the charge of electron and $A=\pi(d / 2)^{2}$ is the area of the sensing surface. Since the sensor sensitivity is defined as $S=\mathrm{d} I / \mathrm{d} c_{\text {bulk }}$, it can be written as

$$
S=\frac{3}{4} \pi D N_{A} C_{e-} \frac{d^{2}}{\Delta z},
$$

as follows from Eqs. (S1-S2). Using the values of $d=30 \mu \mathrm{m}, \Delta z=200 \mu \mathrm{m}$ (Fig. S1), the maximum sensitivity of the combined sensor determined by the physical transport limitation of NO molecules

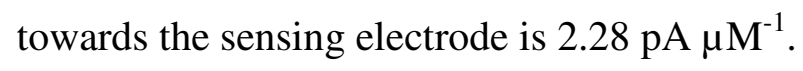




\section{2) Theoretical maximum of sensitivity for a bare single-anode NO-microsensor operating a at constant potential}

In contrast to the combined sensor, the current that can be theoretically reached at a bare single-anode microsensor is not limited by the 1-dimensional diffusive transport of NO molecules through the membrane and electrolyte, but by the 3-dimensional transport of NO molecules from the bulk solution towards the sensing electrode surface. To estimate this flux under constant-potential operation, we assume that the concentration of the NO molecules at the electrode surface is zero and that the transport is diffusive (diffusion coefficient of NO in water of $D=2.21 \times 10^{-9} \mathrm{~m}^{2} \mathrm{~s}^{-1}$ ). To allow analytical expression, we further assume that the sensing area of the electrode is a sphere of radius $r_{0}$ instead of the planar circular area of radius $r_{0}$ (Fig. S2).
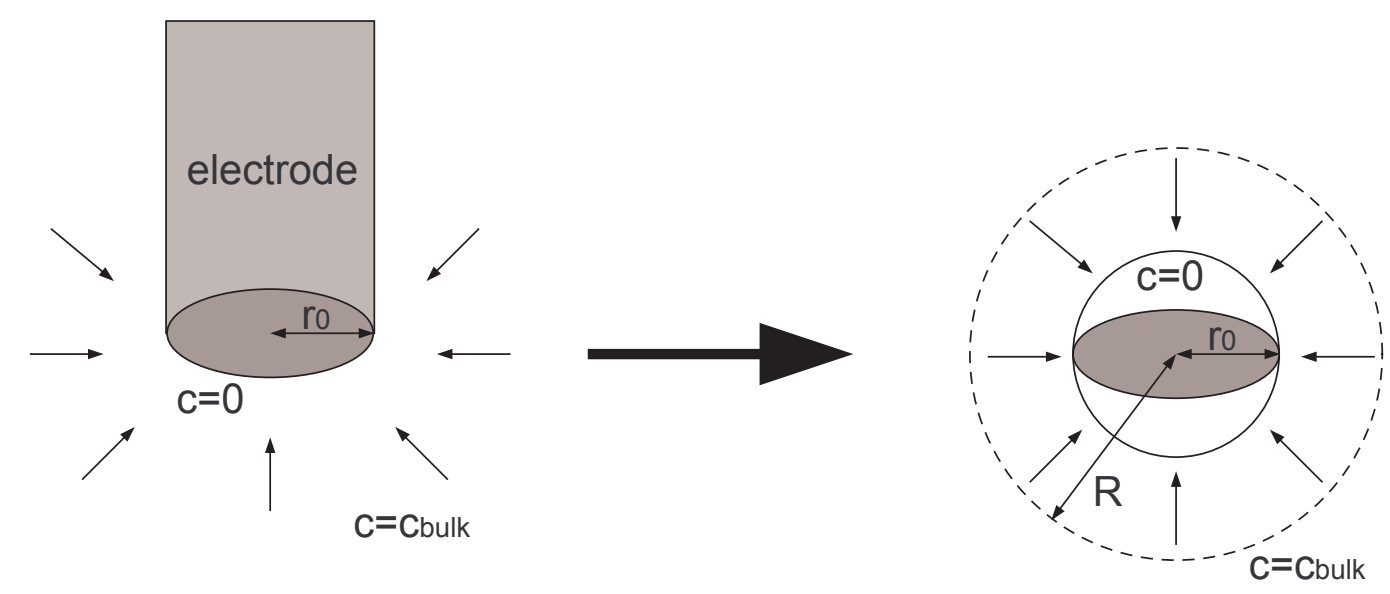

Figure S2:

Consequently, the steady-state concentration field around the sensing surface is spherically symmetric, i.e., dependent only on the radial distance $r$, and fulfills the differential equation 


$$
D \frac{1}{r} \frac{\partial}{\partial r} r^{2} \frac{\partial c}{\partial r}=0
$$

which is the diffusion equation expressed in spherical co-ordinates. The general solution for this differential equation is

$$
c(r)=\frac{A}{r}+B,
$$

where $A$ and $B$ are constants determined from the boundary conditions. The $1^{\text {st }}$ boundary condition is derived from the assumption that the concentration at the spherical sensing surface is zero, i.e.,

$$
c\left(r_{0}\right)=0 \mu M=\frac{A}{r_{0}}+B .
$$

The $2^{\text {nd }}$ boundary condition is derived from the assumption that the concentration in the bulk solution is $c_{\text {bulk }}$, i.e.,

$$
c(r \rightarrow \infty)=B=c_{\text {bulk }} .
$$

Using the general solution (S5), the radial flux can be calculated as

$$
J_{r}=-D \frac{\partial c}{\partial r}=D \frac{A}{r^{2}}
$$

Thus, the NO flux at the sensing surface can be expressed as

$$
J_{r}\left(r_{0}\right)=D \frac{A}{r_{0}^{2}}=-D \frac{B}{r_{0}} .
$$

Considering that each NO molecule oxidized at the sensing surface results in the donation of 3 electrons to the electrochemical circuit, the current through the electrode can be written as

$$
I=3 A_{\text {eff }} J \mathrm{~N}_{\mathrm{A}} \mathrm{C}_{\mathrm{e}-},
$$


where $\mathrm{N}_{\mathrm{A}}=6.022 \times 10^{23} \mathrm{~mol}^{-1}$ is Avogadro's constant, $\mathrm{C}_{\mathrm{e}}=-1.6022 \times 10^{-19} \mathrm{C}$ is the charge of electron and $A_{e f f}=4 \pi r_{0}^{2}$ is the area of the effective sensing surface. The sensor sensitivity is defined as $S=$ $\mathrm{d} I / \mathrm{d} c_{\text {bulk }}$. Thus, combining Eqs. (S6-S10), the maximum sensor sensitivity of a bare single-anode sensor can be approximated as

$$
S=12 \pi D N_{A} C_{e-} r_{0}
$$

which is equal to $120.3 \mathrm{pA} \mu \mathrm{M}^{-1}$ when $r_{0}=15 \mu \mathrm{m}$ (Fig. S2) is used. Thus the sensitivity of a bare single-anode sensor is $\sim 54$ times greater than that for a combined sensor (see above), when the transport of NO molecules towards the sensing surface is diffusive in the entire volume of the bulk medium.

If the bulk medium is stirred, the same model as above can be applied but the concept of a diffusive boundary layer ${ }^{3}$ needs to be additionally taken into account. This is done by considering that the radial distance $\mathrm{R}$ at which the concentration around the sensing surface reaches the bulk value $\mathrm{c}_{\text {bulk }}$ is decreased from infinity (as in the unstirred medium above) to a finite value. This condition leads to the modification of Eq. (S7) representing the second boundary condition, namely

$$
c(R)=\frac{A}{R}+B=c_{b u l k} .
$$

Combining Eqs. (S8), (S10) and (S12), the maximum sensor sensitivity of a bare single-anode sensor in a stirred medium can be approximated as

$$
S=12 \pi D N_{A} C_{e-} r_{0} \frac{R}{R-r_{0}},
$$

which is by a factor of $R /\left(R-r_{0}\right)$ greater than the sensitivity in an unstirred medium. When the diffusive boundary layer is thin, such as during vigorous stirring of the medium, $R$ may become close to $r_{0}$ and the enhancement factor may increase considerably. For example, sensitivity may increase twice to 240 pA $\mu \mathrm{M}^{-1}$ when $R=30 \mu \mathrm{m}$ is used, whereas it can increase 8.5 times to $\sim 1 \mathrm{nA} \mu \mathrm{M}^{-1}$ for $R=17 \mu \mathrm{m}$. 

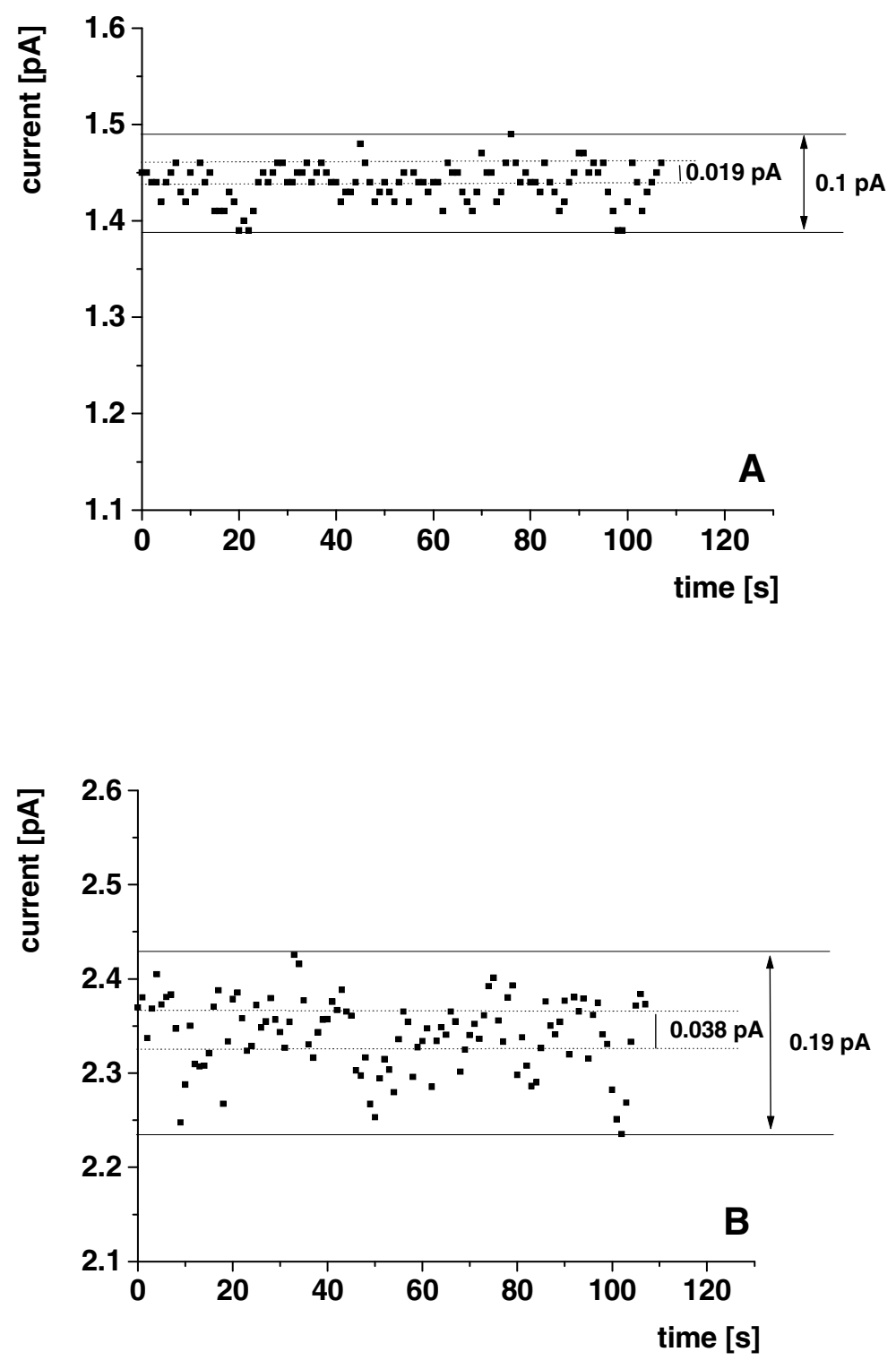

Figure S3. Fluctuations of a background current of a combined NO microsensor (A) with and (B) without a connected guard anode. The sensor was connected with the guard anode for $\sim 1$ month beforehand and currents were recorded after disconnecting the guard anode and after reconnecting the guard anode again. Solid lines represent maximum difference between 107 subsequent recordings, while dotted lines represent their standard deviation. 
(1) Zacharia, I. G.; Deen, W. M. Annals of Biomedical Engineering 2005, 33, 214-222.

(2) Mowery, K. A.; Meyerhoff, M. E. Polymer 1999, 40, 6203-6207.

(3) Dade, W. B., Hogg, A.J. \& Boudreau, B.P. In The Benthic Boundary Layer: Transport

Processes and Biogeochemistry; Boudreau, B. P. J., B.B., Ed.; Oxford University Press: Oxford, 2001. 